\title{
Negative LC3b immunoreactivity in cancer cells is an independent prognostic predictor of prostate cancer specific death
}

\author{
Ashkan Mortezavi ${ }^{1}$, Souzan Salemi ${ }^{1}$, Niels J. Rupp ${ }^{2}$, Jan Hendrik Rüschoff ${ }^{2}$, Thomas \\ Hermanns ${ }^{1}$, Cedric Poyet ${ }^{1}$, Marco Randazzo', Hans-Uwe Simon ${ }^{3}$, Holger Moch ${ }^{2}$, \\ Tullio Sulser ${ }^{1}$, Peter Wild ${ }^{2}$ and Daniel Eberli ${ }^{1}$ \\ ${ }^{1}$ Department of Urology, University Hospital Zurich, University of Zurich, 8091 Zurich, Switzerland \\ ${ }^{2}$ Institute of Surgical Pathology, University Hospital Zurich, University of Zurich, 8091 Zurich, Switzerland \\ ${ }^{3}$ Institute of Pharmacology, Inselspital, University of Bern, 3010 Bern, Switzerland \\ Correspondence to: Daniel Eberli, email: Daniel.Eberli@usz.ch \\ Keywords: autophagy, LC3b, prostate cancer, biochemical recurrence, survival \\ Received: August 29, $2016 \quad$ Accepted: February 20, $2017 \quad$ Published: March 07, 2017 \\ Copyright: Mortezavi et al. This is an open-access article distributed under the terms of the Creative Commons Attribution License \\ (CC-BY), which permits unrestricted use, distribution, and reproduction in any medium, provided the original author and source are \\ credited.
}

\section{ABSTRACT}

Background: Autophagy is a catabolic cellular process used for degradation of cytoplasmic organelles and preservation of cell viability. In this study we aimed to analyse the level of autophagy markers in benign and malignant prostate tissue and to evaluate the prognostic properties for patients with prostate cancer ( $\mathrm{PCa}$ ).

Results: LC3b expression was significantly upregulated in PCa, especially in metastatic and castration-resistant PCa samples compared to benign prostate tissue $(p<0.001)$. Evaluation of expression in malignant radical prostatectomy specimens revealed an inverse association with preoperative serum PSA levels $(p=0.02)$ and Gleason Score $(p=0.07)$. LC3b immunoreactivity was identified as a novel predictor of PCa specific death after radical prostatectomy, independent of Gleason score, tumour stage, and surgical margin status in a multivariable cox regression analysis (hazard ratio $0.09,95 \%$ confidence interval $0.01-0.69, p=0.021$ ). A significant association of ATG-5 and Beclin 1 with LC3b expression could be noticed $(p<0.001)$, but no link with other clincopathologic parameters was observed.

Materials and Methods: A Tissue microarray containing 468 formalin-fixed, paraffin-embedded prostate tissue cores was stained immunohistochemically for major autophagy proteins LC3b, ATG5 and Beclin 1. Immunoreactivity was semiquantitatively scored and correlated with pathologic and clinical parameters, including tumour stage, Gleason score, preoperative PSA level, biochemical recurrence rate and survival. The median clinical follow-up was 132 months.

Conclusion: LC3b was significantly overexpressed in malignant compared to benign prostate tissue. However, positive LC3b immunoreactivity in PCa, as a marker of increased autophagy, was independently associated with a reduced disease-specific mortality.

\section{INTRODUCTION}

When prostate cancer $(\mathrm{PCa})$ is localized in the prostate and considered significant, the treatment of choice is prostatectomy or radiation [1]. In advanced and metastatic PCa androgen ablation is the standard of care to palliate symptoms and postpone cancer related complications [2]. However, after short-term remission, surviving tumour cells reappear as castration-resistant PCa (CRPC) and lead to death within months or few years [3]. Therefore, extensive efforts are made to offer new therapeutic options to patients with CRPC including development of new anti-androgens and combination therapies. One promising approach has been the pharmacological inhibition of autophagy combined with androgen ablation or novel antitumour agents [4]. 
Autophagy is a catabolic cellular mechanism involving degradation of unnecessary or dysfunctional cellular components through autophagosomes allowing maintenance of homeostasis and ensuring cell survival under stress conditions. [5] The process of autophagy is regulated by autophagy-related proteins (ATGs) [6]. The key elements necessary for autophagosome formation are two ubiquitin like conjugation systems: (1) the ATG12ATG5 and (2) the microtubule-associated protein 1 light chain 3 (LC3)-phosphatidylethanolamine systems [7]. In mammalian cells, three types of LC3 were reported; $\mathrm{A}, \mathrm{B}$ and $\mathrm{C}$, with $\mathrm{LC} 3 \mathrm{~b}$ expression being the most valid marker of autophagosome formation [8] and therefore one of the most widely used in situ techniques of autophagy measurement in benign and malignant tissue [9].

Whether autophagy acts as a promoter or a suppressor during tumorigenesis seems to be context and organ-specific. Improvement of the cytotoxic effect of chemotherapy drugs or resensitization of chemoresistent tumour cells through inhibition of the protective role of autophagy has been reported in several studies [10]. Particularly in PCa, targeting autophagy is a promising new therapeutic modality [1117]. However, the relevance of autophagy-related protein expression in treatment-naive PCa is largely unknown.

In the current study, we analyse the expression profile of the three main autophagy markers involved in autophagosome formation, LC3b, ATG5 and Beclin 1 in benign prostate tissue, localized $\mathrm{PCa}, \mathrm{CRPC}$ and $\mathrm{PCa}$ metastases, respectively. Autophagy marker expression is further correlated with clinicopathological parameters including biochemical recurrence free survival (BCRFS), overall- (OS) and disease-specific survival (DSS).

\section{RESULTS}

\section{Descriptive analysis}

Clinicopathological characteristics of the patients are summarized in Table 1. Men in the NADT group had higher mean PSA level $(32.7 \pm 57.4 \mathrm{ng} / \mathrm{ml}$ vs. 15.6 $\pm 19.3 \mathrm{ng} / \mathrm{ml},<0.001)$ and more advanced tumours (pT3/4 tumours in $56.4 \%$ vs. $32.4 \%, \mathrm{p}=0.002$ ).

A total of $420 / 468(89.7 \%)$ cores could be evaluated for LC3b, 429/468 (91.7\%) for Beclin 1 and 417/468 (89.1\%) for ATG5 immunostaining. Representative stainings are summarized in Figure 1. In general, positive expression of LC $3 b$, Beclin 1 and ATG5 was detectable in 124 of 420 (29.5\%), 259 of $429(55.3 \%)$ and 287 of 417 (68.8\%) of analysable cases, respectively. Immunoreactivity for LC3b and Beclin 1 was low in benign prostatic tissue (BPH, $2.5 \%$ and $24.4 \%, \mathrm{p}<0.001$, Figure 2). LC 3b and Beclin 1 expressions were significantly increased in primary malignant RP specimen (36.1\% and 65.9\%, p<0.001), in CRPC (34.6\% and $62.5 \%, \mathrm{p}<0.001$ ) and in metastatic tissue (MTS, 33.3\% and $53.1 \%, \mathrm{p}<0.001)$. Contrary, ATG5 showed a higher expression in benign tissue compared to malignant tissue $(\mathrm{p}=0.006)$. NADT tissue showed a similar immunoreactivity for Beclin 1 and ATG5 when compared to primary RP. However, the expression of LC3b was significantly lower in these men when compared to treatment naïve tissue $(11.5 \%$ vs. $36.1 \%, p<0.001)$. LC3b expression was higher in in local lymph node metastases compared to distant locations (e.g. liver, lung, bone; $46.7 \%$ vs. $23.8 \%, \mathrm{p}=0.17$ ).

Regarding all tissue samples, positive LC3b immunostaining was highly associated with positive Beclin 1 and ATG5 immunostaining $(\mathrm{p}<0.001$, Table 2$)$. Negative immunoreactivity of Beclin 1 and ATG5 was associated in $89 \%$ and $96 \%$ of the cases with a lack of LC3b staining, respectively $(\mathrm{p}<0.001)$. However, a positive immunostaining for Beclin 1 and ATG5 was related to a LC3b positive staining in only $41 \%$ and $47 \%$ of the cases, respectively ( $p<0.001$ ).

Clinicopathologic characteristics of RP patients were correlated with LC3b, Beclin 1 and ATG5 expression (Table 1). In primary $\mathrm{PCa}$, patients with a negative immunoreactivity for LC3b had higher preoperative PSA levels $(p=0.02)$ and higher Gleason scores $(p=0.07)$. No associations could be found for Beclin 1 and ATG5 with clinicopathological parameters.

After exclusion of noninterpretable tissue samples $(\mathrm{n}=26)$ and patients who had received NADT $(\mathrm{n}=56)$ a total of 266 RP patients were considered for survival analysis. Postoperative PSA values were available for 235 patients $(88.3 \%)$ with 58 not reaching a nadir of $<0.1 \mathrm{ng} / \mathrm{ml}$ (e.g. 177 included for BCRFS analysis). A total of 55 patients (31.1\%) experienced a BCR during the follow-up period (30 LC3b negative, 25 LC3b positive). Clinical follow-up data for evaluation of the OS and DSS rate was available for 241 patients $(90.1 \%)$. Fifty patients died of any cause with 19 due to PCa (17 LC3b negative, 2 LC3b positive).

\section{Survival analysis}

Univariate Cox regression analysis (Table 3) and log-rank statistics showed no association of LC3b (Figure 3A), Beclin 1 and ATG5 staining with BCRFS, in contrast to the established parameters like Gleason score, surgical margin status and tumour stage. However, positive immunoreactivity for LC3b was highly associated with longer OS (hazard ratio [HR] 0.48, 95\% confidence interval [CI] 0.24-0.95, $\mathrm{p}=0.034$, Figure $3 \mathrm{~B}$ ) and DSS (HR 0.09, CI 0.01-0.71, $\mathrm{p}=0.022$, Table 4 and Figure $3 \mathrm{C}$ ). The estimated 15 -year DSS rate was $100 \%$ for LC 3 bpositive cases and $87 \%$ for LC3b-negative tumours, respectively $(\mathrm{p}=0.003)$. In a subgroup analysis of patients with moderately differentiated tumours (Gleason score 7), negative LC3b immunoreactivity was associated with shorter DSS times $(n=145, \log$ rank $p=0.04)$. Beclin 1 and ATG5 immunostaining were not associated with shorter DSS.

Multivariable Cox regression analysis was developed for the assessment of the DSS rate. Characteristics of variables are shown in Table 4. Because 
Table 1: Clinicopathological characteristic in relation to LC3b, ATG5 and Beclin 1 immunoreactivity in radical prostatectomy specimens (patients with neoajuvant androgen deprivation therapy were not considered)

\begin{tabular}{|c|c|c|c|c|c|c|c|c|c|}
\hline \multirow{2}{*}{$\begin{array}{l}\text { Variable and } \\
\text { Characteristics }\end{array}$} & \multicolumn{3}{|c|}{ LC3b Immunoreactivity } & \multicolumn{3}{|c|}{ ATG5 Immunoreactivity } & \multicolumn{3}{|c|}{ Beclin 1 Immunoreactivity } \\
\hline & Negative & Positive & $\mathbf{P}$ & Negative & Positive & $\mathbf{P}$ & Negative & Positive & $\mathbf{P}$ \\
\hline \multicolumn{10}{|l|}{ Age at diagnosis } \\
\hline years (SD) & $63.8( \pm 6)$ & $62.9( \pm 6)$ & 0.27 & $64.3( \pm 6)$ & $63.2( \pm 6)$ & 0.15 & $63.4( \pm 6)$ & $63.6( \pm 6)$ & 0.79 \\
\hline \multicolumn{10}{|l|}{ PSA at diagnosis } \\
\hline ng/ml (SD) & $16.7( \pm 18)$ & $12.2( \pm 10)$ & 0.02 & $20.3( \pm 31)$ & $13.5( \pm 11)$ & 0.08 & $13.5( \pm 13)$ & $16.4( \pm 22)$ & 0.28 \\
\hline \multicolumn{10}{|c|}{ Gleason Score (\%) } \\
\hline $5-6$ & $26(51)$ & $25(49.0)$ & 0.07 & $12(22.6)$ & $41(77.4)$ & 0.21 & $17(32.1)$ & $36(67.9)$ & 0.95 \\
\hline 7 & $106(65.0)$ & $57(35.0)$ & & $51(30.9)$ & $114(69.1)$ & & $58(33.9)$ & $113(66.1)$ & \\
\hline $8-10$ & $37(72.5)$ & $14(27.5)$ & & $20(38.5)$ & $32(61.5)$ & & $19(35.2)$ & $35(64.8)$ & \\
\hline \multicolumn{10}{|l|}{ Tumor stage (\%) } \\
\hline pT2 & $108(61.7)$ & $67(38.3)$ & 0.34 & $52(28.6)$ & $130(71.4)$ & 0.21 & $70(37.0)$ & $119(63.0)$ & 0.13 \\
\hline $\mathrm{pT} 3 / 4$ & $61(67.5)$ & $28(31.5)$ & & $32(36.4)$ & $45(63.6)$ & & $24(27.3)$ & $64(72.7)$ & \\
\hline \multicolumn{10}{|c|}{ Surgical margins (\%) } \\
\hline negative & $113(65.3)$ & $60(34.7)$ & 0.34 & $56(31.3)$ & $123(68.7)$ & 1.00 & $66(35.9)$ & $118(64.1)$ & 0.34 \\
\hline positive & $52(59.1)$ & $36(40.9)$ & & $27(31.0)$ & $60(69.0)$ & & $27(30.0)$ & $63(70.0)$ & \\
\hline \multicolumn{10}{|l|}{ Nodal status (\%) } \\
\hline pN0 & $134(66.0)$ & $69(34.0)$ & 0.11 & $65(31.4)$ & $142(68.6)$ & 0.51 & $74(34.9)$ & $138(65.1)$ & 1.00 \\
\hline $\mathrm{pN} 1$ & $10(90.9)$ & $1(9.1)$ & & $2(18.2)$ & $9(81.8)$ & & $4(36.4)$ & $7(63.6)$ & \\
\hline \multicolumn{10}{|c|}{ Metastasis during follow-up* $(\%)$} \\
\hline no & $107(59.8)$ & $72(40.2)$ & 0.02 & $57(31.0)$ & $127(69.0)$ & 1.00 & $64(33.5)$ & $127(66.5)$ & 0.15 \\
\hline yes & 25 (80.6) & $6(19.4)$ & & $10(32.3)$ & $21(67.7)$ & & $15(48.4)$ & $16(51.6)$ & \\
\hline
\end{tabular}

Continously coded variables are given with mean standard deviation (SD). Bold face reprensenting $\mathrm{P}<0.05$.

* Minimum follow-up of 5 years.

of model assumptions (noninformative censoring, proportional hazards), only LC3b expression, Gleason score, tumour stage and surgical margin status were considered. Positive LC3b immunoreactivity (HR 0.09, CI $0.01-0.69, \mathrm{p}=0.021$ ), higher Gleason score (HR 3.48, CI 1.23-9.88, $\mathrm{p}=0.019$ ) and higher tumour stage (HR 2.23, CI 1.16-4.29, $\mathrm{p}=0.017)$ remained independent prognostic parameters for a $\mathrm{PCa}$ specific death.

\section{DISCUSSION}

This is the first study investigating expression levels of the established markers of autophagy LC3b, Beclin 1 and ATG5 and their association with the solid clinical endpoint of survival in a representative cohort of PCa patients. LC3b, a major marker of autophagy, was significantly upregulated in adenocarcinomas of the prostate, in metastatic and in CRPC. Within the group of patients with localized $\mathrm{PCa}$, who underwent radical prostatectomy, the absence of LC3b immunoreactivity was identified as a novel predictor of PCa-specific death after radical prostatectomy, which was independent of the well-established predictive factors Gleason score, tumour stage, and surgical margin status.

Autophagy not only plays a central role in maintenance of cellular homeostasis, but also is essential in the process of malignant transformation [18]. The pro-survival and pro-death role of autophagy can differ, depending on several different factors such as tissue type, stimulating factors and cellular environment [19]. Accumulating evidence support the thesis, that autophagy is a tumour suppressor and inhibits tumour development by detaching damaged organelles and promoting cell death of cancerous cells [20]. Particularly, downregulation of ATG5 contributed to tumorigenesis of earlystage cutaneous melanoma in a study by Liu et al. [21]. 
Benign tissue
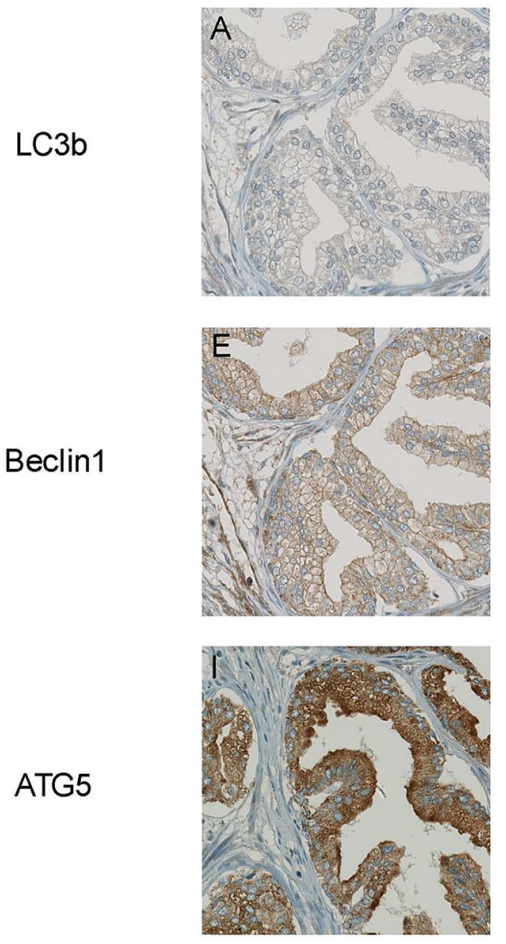

RPE
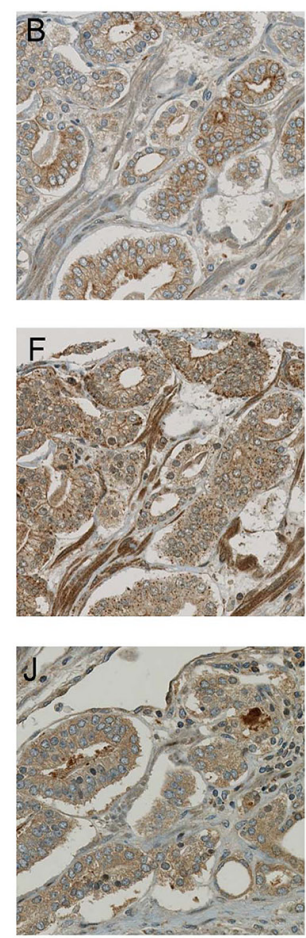

CRPCa
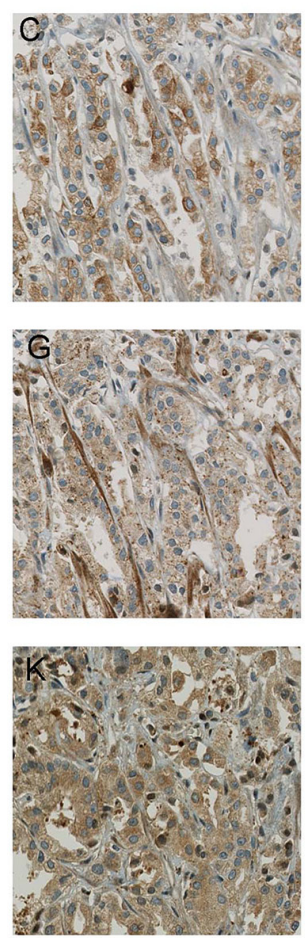

LN Metastasis
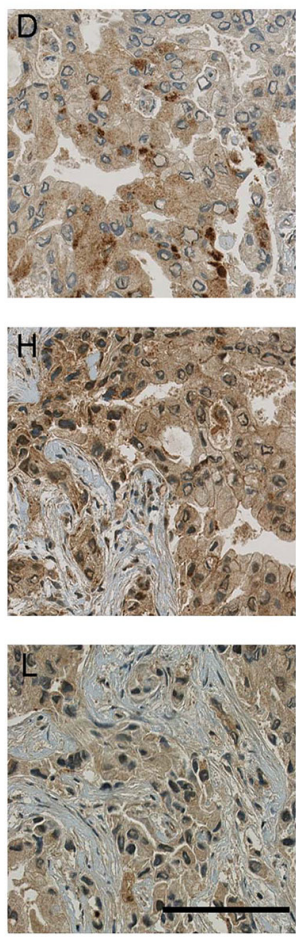

Figure 1: Representative images of benign prostatic tissue and three different cancer tissues. Normal tissue showed negative LC3b (A), weak Beclin 1 (E) and strong ATG5 (I) immunoreactivity. In a radical prostatectomy case (RP) with acinar adenocarcinoma stronger positivity for LC3b (B) and Beclin 1 (F) was observed. In contrast, ATG5 displayed weaker expression levels (J) compared to the respective benign tissue. Similar staining results were found in castration resistant prostate cancer cases (CRPC; $\mathbf{C}, \mathbf{G}$ and $\mathbf{K})$ and a lymph node metastasis (D, $\mathbf{H}$ and $\mathbf{L})$. Scale bar $100 \mu \mathrm{m}$.
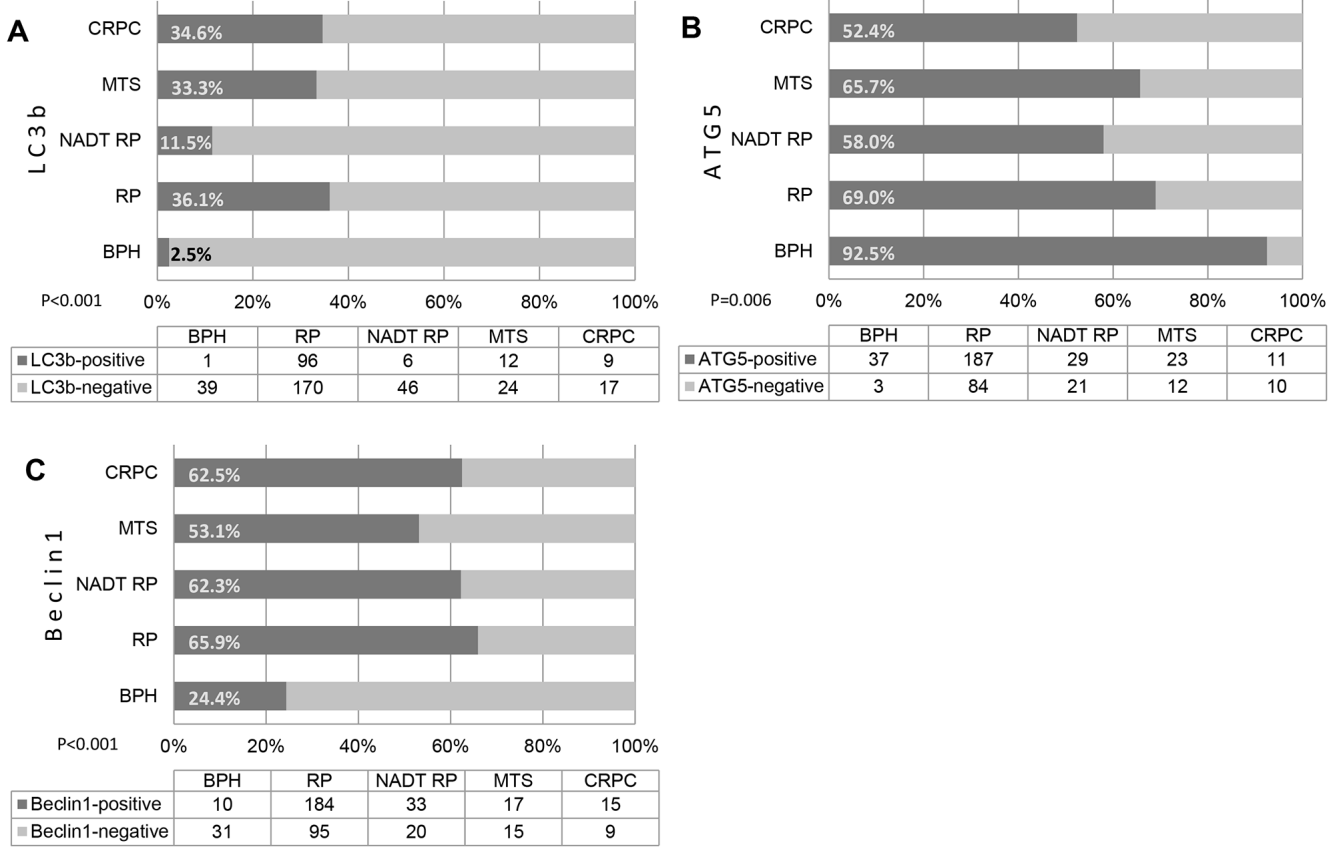

Figure 2: A-C, Cumulative bar chart representing immunoreactivity for LC3b (A), ATG5 (B) and Beclin 1 (C) in different tissue types. BPH, benign prostate hyperplasia. Bars represent percentage of positive and negative cases. Table below contains number of patients in each group. RP, radical prostatectomy (malignant); NADT RP, neoadjuvant androgen deprivation therapy (RP specimen); MTS, prostate cancer metastasis; CRPC, castration-resistant prostate cancer. 
Table 2: Correlation between cytoplasmatic immunoreactivity of LC3b, ATG5 and Beclin 1 in all tissue samples

\begin{tabular}{|c|c|c|c|c|c|c|c|c|c|}
\hline & \multicolumn{3}{|c|}{ LC3b Immunoreactivity } & \multicolumn{3}{|c|}{ ATG5 Immunoreactivity } & \multicolumn{3}{|c|}{ Beclin 1 Immunoreactivity } \\
\hline & Negative & Positive & $\mathbf{P}$ & Negative & Positive & $\mathbf{P}$ & Negative & Positive & $\mathbf{P}$ \\
\hline \multicolumn{10}{|l|}{ LC3b } \\
\hline Negative & & & & $114(41.5)$ & $161(58.5)$ & $<0.001$ & $156(54.7)$ & $129(45.3)$ & $<0.001$ \\
\hline Positive & & & & $13(10.4)$ & $112(89.6)$ & & $7(5.6)$ & $117(94.4)$ & \\
\hline \multicolumn{10}{|l|}{ ATG5 } \\
\hline Negative & $114(89.8)$ & $13(10.2)$ & $<0.001$ & & & & $75(60.0)$ & $50(40.0)$ & $<0.001$ \\
\hline Positive & $161(59.0)$ & $112(41.0)$ & & & & & $88(32.0)$ & $187(68.0)$ & \\
\hline \multicolumn{10}{|l|}{ Beclin 1} \\
\hline Negative & $156(95.7)$ & $7(4.3)$ & $<0.001$ & $70(46.0)$ & $88(54.0)$ & $<0.001$ & & & \\
\hline Positive & $129(52.4)$ & $117(47.6)$ & & $50(21.1)$ & $187(78.9)$ & & & & \\
\hline
\end{tabular}

Note: Bold face reprensenting $\mathrm{P}<0.05$.

Table 3: Univariable Cox regression analysis für recurrence-free survival

\begin{tabular}{lcccc}
\hline Variable & Characteristics** & \multicolumn{3}{c}{ Recurrence-free survival } \\
\cline { 3 - 5 } & & HR & $\mathbf{9 5 \%}$ CI & P* \\
\hline Age at diagnosis & years & 1.01 & $0.97-1.06$ & 0.527 \\
Gleason score (grouped) & $5-7$ vs. 7 vs. 8-10 & 2.27 & $1.49-3.45$ & $<\mathbf{0 . 0 0 1}$ \\
Preoperative PSA level & ng/mL & 1.01 & $0.99-1.01$ & 0.284 \\
Surgical margin status & negative vs positive & 3.00 & $1.82-4.94$ & $<\mathbf{0 . 0 0 1}$ \\
Tumor stage (pT) & pT2 vs. pT3a vs. pT3b/4 & 2.03 & $1.46-2.83$ & $<\mathbf{0 . 0 0 1}$ \\
LC3b & negative vs positive & 0.76 & $0.47-1.3$ & 0.307 \\
ATG5 & negative vs positive & 1.00 & $0.60-1.66$ & 0.993 \\
Beclin 1 & negative vs positive & 1.19 & $0.74-1.91$ & 0.468 \\
\hline
\end{tabular}

* Bold face reprensenting $\mathrm{P}<0.05$.

** left category used as a reference

vs, versus

Deletions of Beclin 1 have been described in specimens of human breast, ovarian and prostate tumours [22, 23]. On the other hand, other studies reported that increased autophagy can support cancer development by maintaining the stability of intracellular environment and acts as a protective mechanism against apoptosis and external death stimuli including anti-cancer drugs [24]. Evidence for a role of both reduced and increased autophagy in cancer cells was also found in the present study; while higher expression of autophagy proteins were observed in malignant tissue, lack of autophagy proteins was associated with worse clinicopathological parameters and with an increased tumour specific mortality.

Our findings are in contrast with a recently published report investigating the immunohistochemical expression of LC3b and Beclin 1 in 96 PCa specimens [25]. Giatromanolaki et al. found a significant association of high Gleason scores and high tumour stages with high LC3b and Beclin 1 expression levels. However, this report exclusively included node-negative $\mathrm{PCa}$ of which $68 \%$ had a Gleason score of $\leq 6$. Only 31 specimens with a Gleason score $\geq 7$ and 19 with an extracapsular extension were evaluated compared to 214 with Gleason score $\geq 7$ (including 51 Gleason 8-10) and 81 with a tumour stage $\geq$ pT3 in the present study. Furthermore, due to the pooling of moderately differentiated tumours of Gleason 7 with the poorly differentiated of Gleason 8-10 it remains unclear, how many genuine high risk tumours were stained in this study cohort. No clinical data regarding preoperative PSA values, patterns of biochemical recurrences or survival 
rates were available. To further validate our results, we analysed publicly available TCGA expression data and observed alterations of LC3b mRNA (MAP1LC3B) in 70 (14\%) of 498 cases. LC3b mRNA alterations (mainly downregulations) were associated with a significantly shorter disease-free survival ( $p=0.0219$, data not shown).

Liu et al. just recently evaluated the prognostic impact of 5 autophagy markers on the clinical endpoint of BCR after radical prostatectomy and reported no association for $\mathrm{LC} 3 \mathrm{~b}$ in agreement with our results. Yet, an association of positive UNC-51-like kinase 1 (ULK1) reactivity with BCR was reported after a relatively short median follow-up of 51 months [26]. In the present study, no association of autophagy with BCR rates could be observed. However, after a median follow-up of over 10 years we could show that a positive LC3b immunoreactivity had a positive and distinct impact on overall and disease-specific survival. This impact remained significant in a multivariable analysis with a $\mathrm{HR}$ of 0.09 (95\% CI 0.01-0.69) for dying of PCa if the staining for LC $3 \mathrm{~b}$ showed an expression at the time of prostatectomy. LC3b immunoreactivity was an even more powerful predictor of PCa specific death than the well-established parameters Gleason score and tumour stage. However, the low number of prostate cancer specific deaths lead to a wide $95 \%$ CI in our model with a significant influence on the point estimator (0.09). Considering HRs of other biomarkers, the real HR is expected to be slightly higher (95\% CI 0.01-0.69) without having an impact on the conclusions drawn from our analysis.
Patients who had received ADT prior to surgery were excluded from the primary analysis in order to minimize the confounding effect of a neoadjuvant treatment on protein expression. However, this population can be used to provide an insight on the effect of ADT on autophagy. Recently, several studies have investigated the role of autophagy as an escape mechanism of PCa exposed to antitumour agents, hormonal ablation and radiation therapy $[4,27,28]$. An inhibition of autophagy was able to increase the antitumour effect of these agents $[12,14]$. Of note, inhibition of autophagy in androgen sensitive cell lines (e.g. LNCaP) under hormonal ablation therapy showed reduced cell viability, suggesting the protective role of autophagy in androgen withdrawal [16, 17]. We, therefore, expected an up-regulation of autophagy in hormonal naïve patients treated with androgen withdrawal prior to surgery. Our findings demonstrated the opposite; autophagy was significantly reduced in our cohort of patients $(11.5 \%$ vs. $36.1 \%, \mathrm{p}<0.001)$ compared to the control group. However, preoperative ADT was mainly given to patients with high-risk disease and, therefore, this subgroup of patients differed in its perioperative parameters significantly from the control group.

Based on the methods of this investigation, no conclusions can be drawn regarding the causal relation of these findings. However, our results provide a new insight into the role of autophagy in $\mathrm{PCa}$ patients and indicate that the regulation of autophagy in $\mathrm{PCa}$ depends on the stage of the tumour. Increased autophagy levels may be a sign of starvation and metabolic stress in well
A
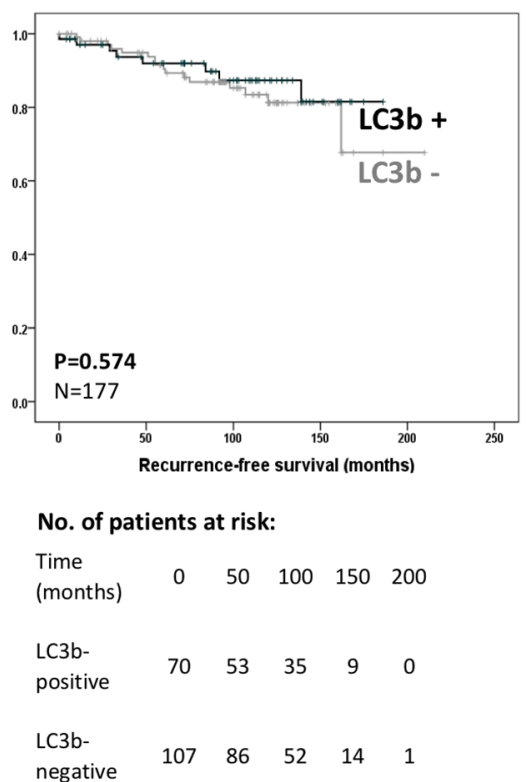

B

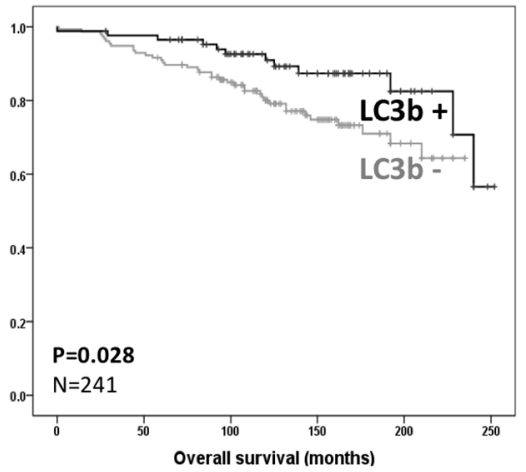

No. of patients at risk:

$\begin{array}{llllll}\text { Time } & 0 & 50 & 100 & 150 & 200\end{array}$

$1 \mathrm{C} 3 \mathrm{~b}-$

$\begin{array}{llllll}\text { positive } & 86 & 83 & 67 & 44 & 11\end{array}$

$\begin{array}{llllll}\text { LC3b- } & 155 & 144 & 116 & 63 & 19\end{array}$
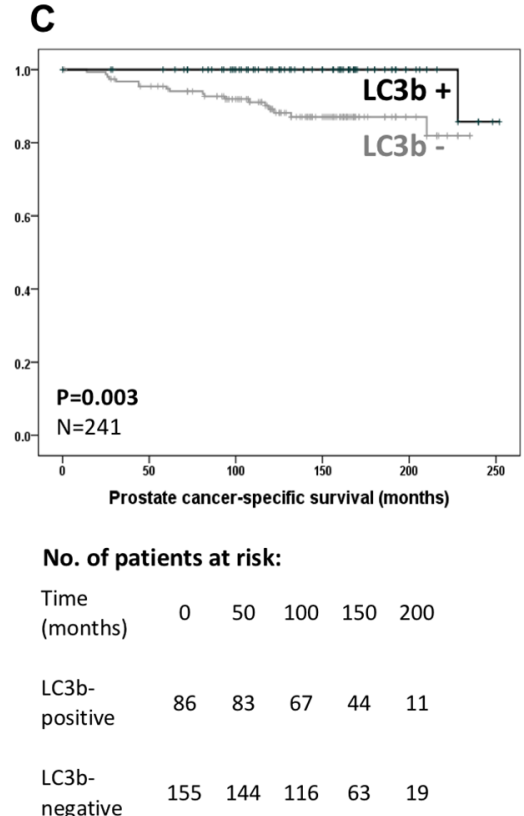

Figure 3: Kaplan-Meier curves regarding biochemical recurrence-free. (A), overall (B) and disease-specific (C) survival in patients with clinically localized prostate cancer and negative LC3b (LC3b -) versus positive LC 3b immunoreactivity $(\mathrm{LC} 3 \mathrm{~b}+)$. 
Table 4: Uni- (A) and multivariable (B) Cox regression analysis for tumor-specific survival

\begin{tabular}{|c|c|c|c|c|}
\hline \multicolumn{5}{|l|}{ A. Univariable } \\
\hline \multirow[t]{2}{*}{ Variable } & \multirow[t]{2}{*}{ Characteristics** } & \multicolumn{3}{|c|}{ Prostate cancer-specific survival } \\
\hline & & HR & $95 \%$ CI & $P^{*}$ \\
\hline Age at diagnosis & Years & 1.06 & $0.97-1.16$ & 0.177 \\
\hline Gleason score (grouped) & $5-6$ vs. 7 vs. $8-10$ & 4.70 & $2.16-10.23$ & $<0.001$ \\
\hline Preoperative PSA level & $\mathrm{ng} / \mathrm{mL}$ & 1.01 & $0.99-1.02$ & 0.171 \\
\hline Surgical margin status & negative vs. positive & 2.35 & $1.0-5.54$ & 0.050 \\
\hline Tumor stage (pT) & pT2 vs. pT3a vs. pT3b/4 & 3.15 & $1.90-5.2$ & $<0.001$ \\
\hline $\mathrm{LC} 3 \mathrm{~b}$ & negative vs. positive & 0.09 & $0.01-0.71$ & 0.022 \\
\hline ATG5 & negative vs. positive & 0.60 & $0.24-1.53$ & 0.287 \\
\hline Beclin 1 & negative vs. positive & 1.05 & $0.41-2.6$ & 0.924 \\
\hline \multicolumn{5}{|l|}{ B. Multivariable } \\
\hline Gleason score (grouped) & $5-6$ vs. 7 vs. $8-10$ & 3.48 & $1.23-9.88$ & 0.019 \\
\hline Surgical margin status & negative vs. positive & 1.34 & $0.48-3.69$ & 0.576 \\
\hline Tumor stage (pT) & pT2 vs. pT3a vs. pT3b/4 & 2.23 & $1.16-4.29$ & 0.017 \\
\hline $\mathrm{LC} 3 \mathrm{~b}$ & negative vs. positive & 0.09 & $0.01-0.69$ & 0.021 \\
\hline
\end{tabular}

* Bold face reprensenting $\mathrm{P}<0.05$.

** left category used as a reference

vs, versus

differentiated tumours due to poorer selective advantage. In more aggressive tumours, cells may have activated other mechanisms to deal with cellular stress and nutrition deficiency making them independent from autophagy proteins. Therefore, low or no autophagy might be a sign of malignant cells not struggling with survival. Further investigations will be necessary evaluating the actual functional role of autophagy in different stages of PCa.

\section{MATERIALS AND METHODS}

\section{Patients and specimen characteristics}

Tissue microarrays (TMA) contained 468 formalin-fixed, paraffin-embedded prostate tissues and were constructed as previously described [29]. Specimens were collected between 1993 and 2007 from the Institute of Surgical Pathology, University Hospital of Zurich, Switzerland. The TMA included a series of 348 consecutive (non-selected) malignant radical prostatectomy (RP) specimens, 29 CRPC samples, 18 lymph node metastases, 28 distant metastases (bone, lung, urinary bladder), and 45 benign prostatic hyperplasia samples. A neoadjuvant androgen deprivation therapy (NADT) had been established preoperatively in 56 men with primary $\mathrm{PCa}$. Haematoxylin and eosin-stained slides of all specimens were re-evaluated by two experienced pathologists (P.J.W., H.M.) to identify representative areas. Tumour stage and Gleason score of the Zurich cohort were assigned according to the International Union Against Cancer and World Health Organization/ International Society of Urological Pathology criteria [30]. In total, clinical follow-up data were available for 317 of 348 prostatectomy patients $(91.1 \%)$. After RP, follow-up of patients was conducted by periodic measurement of the serum PSA. Median follow-up was 132 months (range 1-252). The study was approved by the local scientific ethics committees (KEK-ZH-No.2008-0025).

\section{Immunohistochemical assay}

Consecutive $3-\mu \mathrm{M}$ sections were cut from the TMA tissue blocks. The expression of Beclin 1, ATG5 and LC3b was analysed immunohistochemically using the following primary antibodies: anti-Beclin 1 (rabbit polyclonal; Novus Biologicals, LLC; no. NB110-87318, dilution 1:200), anti-ATG5 (rabbit polyclonal; SigmaAldrich Corporation; no. A0731, dilution 1:1000) and anti-LC3b (rabbit polyclonal; Abcam plc; no. ab48394, dilution 1:200). Beclin 1 and ATG5 stainings were detected by Discovery UltraMap anti-rabbit IgG in combination with ChromoMap DAB Detection Kit (Ventana Medical Systems, Inc.) and LC3b by UltraView Universal DAB Detection Kit (Ventana). After antigen 
retrieval (microwave oven for 10 minutes at $250 \mathrm{~W}$ ) immunohistochemistry for Beclin 1 and ATG5 was carried out in a Discovery Ultra stainer (Ventana) and for LC $3 b$ in a Benchmark Ultra stainer (Ventana) according to the manufacturer's instructions. Normal prostatic and testicular parenchyma was chosen as positive control. For negative controls, the primary antibody was omitted. The specificity of the commercial antibodies has been thoroughly validated in former studies [31-34].

\section{Antibody specificity test}

The specificity of the anti-LC3b (rabbit polyclonal; Abcam plc; no. ab48394, dilution 1:200) antibody was tested using LnCaP prostate cancer cells (American Type Culture Collection, Manassas, VA). LnCaP cells were cultured in the presence of pharmacological modulator of autophagy, $5 \mathrm{mM}$ 3-MA (Sigma-Aldrich, Buchs, Switzerland), an inhibitor commonly used to block autophagy [35] and $2 \mu \mathrm{M}$ rapamycin (LuBioScience, Luzern, Switzerland) an inducer of autophagy for 7 days on the slide flasks (Nunc flasks, cat: 170920). Cells were immunostained with anti-LC3b $(1: 200)$ for $24 \mathrm{~h}$ at $4^{\circ} \mathrm{C}$. The slides were incubated with secondary antibody $\mathrm{Cy} 3-$ conjugated sheep anti-rabbit antibody (Sigma, 1:200) at room temperature for $1 \mathrm{~h}$. The slides were counter-stained with DAPI (4',6-diamidino-2-phenylindole, Sigma, 1:200) and analysed with a Leica fluorescence microscope (40x). The specificity of the anti-LC3b antibody could be confirmed by detecting no staining in the negative control, a minimal staining in cells inhibited by $3 \mathrm{MA}$, a modest staining in untreated cells (basal autophagy level) and the typical LC3b punctuation in cells induced by rapamycin (Supplementary Figure 1).

\section{Immunohistochemical readout}

One surgical pathologist (N.J.R.) and one urologist experienced in TMA readouts (A.M.) performed a blinded evaluation of the slides without knowledge of clinical data. In case of a discrepancy the core was assessed by a senior pathologist (P.J.W.). Causes of noninterpretable results included lack of target tissue, presence of necrosis, or crush artefact. Since autophagy proteins showed a homogeneous expression pattern, staining intensity (exclusively cytoplasmic staining pattern was evaluated) was assigned using a semiquantitive, four-tired score: negative (0), weak $(1+)$, moderate $(2+)$ or strong $(3+)$. In cases with heterogeneity, the predominant staining intensity $(>80 \%)$ was counted. Searching for cut-offs in an unbiased way is a major problem in immunohistochemical studies dealing with a continuous readout. The median LC3b immunoreactivity in prostatectomy cases with malignant tissue (weak) was chosen as cut-off. Accordingly, positive LC3b, ATG5 and Beclin 1 immunoreactivity was defined as moderate or strong staining of target cells.

\section{Statistical analyses}

SPSS version 22.0 (IBM Corporation, Armonk, NY, USA) was used for statistical analyses. To study statistical associations between clinicopathologic and immunohistochemical data, contingency table analysis with 2-sided Fisher's exact tests were used for categorical and the student t-test for continuous variables. Descriptive statistical analyses for patients who had received any hormonal manipulation preoperatively (NADT) were performed separately. Only patients who were treated by radical prostatectomy were considered for survival analysis. The outcome measures were biochemical recurrence-free survival (BCRFS), overall survival (OS) and disease-specific survival (DSS). Biochemical recurrence (BCR) was defined as PSA value $\geq 0.1 \mathrm{ng} /$ $\mathrm{mL}$ with subsequent confirmation after reaching the PSA nadir of $0.1 \mathrm{ng} / \mathrm{ml}$ postoperatively. Patients not reaching this nadir threshold postoperatively were excluded from BCRFS analysis. Univariate Cox regression analysis was performed for BCRFS and DSS. Investigated variables were age, Gleason score, preoperative PSA level, pathologic tumour stage, surgical margin status, immunoreactivity for LC3b, ATG5 and Beclin 1. A stepwise multivariable Cox regression model was adjusted, testing the independent prognostic relevance of LC3b immunoreactivity for DSS. BCRFS, OS and DSS curves were calculated using the Kaplan-Meier method with significance evaluated by 2 -sided log-rank statistics. All p-values $<0.05$ were considered statistically significant.

\section{ACKNOWLEDGMENTS}

We kindly thank Marian Severin Wettstein for assistance with methodology and statistical support. The mRNA results shown in the discussion section here are based upon data generated by the TCGA Research Network: http://cancergenome.nih.gov.

\section{CONFLICTS OF INTEREST}

The authors declare no conflict of interest.

\section{GRANT SUPPORT}

This study was supported by „Fonds zur Förderung des akademischen Nachwuchses" (Research Talent Development Fund, FAN, University of Zurich).

\section{REFERENCES}

1. Heidenreich A, Bastian PJ, Bellmunt J, Bolla M, Joniau S, van der Kwast T, Mason M, Matveev V, Wiegel T, Zattoni F, Mottet N, European Association of U. EAU guidelines on prostate cancer. part 1: screening, diagnosis, and local 
treatment with curative intent-update 2013. Eur Urol. 2014; 65:124-137.

2. Heidenreich A, Bastian PJ, Bellmunt J, Bolla M, Joniau S, van der Kwast T, Mason M, Matveev V, Wiegel T, Zattoni F, Mottet $\mathrm{N}$ and European Association of U. EAU guidelines on prostate cancer. Part II: Treatment of advanced, relapsing, and castration-resistant prostate cancer. Eur Urol. 2014; 65:467-479.

3. Isbarn H, Boccon-Gibod L, Carroll PR, Montorsi F, Schulman C, Smith MR, Sternberg CN , Studer UE. Androgen deprivation therapy for the treatment of prostate cancer: consider both benefits and risks. Eur Urol. 2009; $55: 62-75$.

4. Ziparo E, Petrungaro S, Marini ES, Starace D, Conti S, Facchiano A, Filippini A, Giampietri C. Autophagy in prostate cancer and androgen suppression therapy. International journal of molecular sciences. 2013; 14:12090-12106.

5. Mizushima N, Komatsu M. Autophagy: renovation of cells and tissues. Cell. 2011; 147:728-741.

6. Levine B, Klionsky DJ. Development by self-digestion: molecular mechanisms and biological functions of autophagy. Developmental cell. 2004; 6:463-477.

7. Yang Z, Klionsky DJ. Eaten alive: a history of macroautophagy. Nature cell biology. 2010; 12:814-822.

8. Klionsky DJ and Emr SD. Autophagy as a regulated pathway of cellular degradation. Science. 2000; 290:1717-1721.

9. Miracco C, Cevenini G, Franchi A, Luzi P, Cosci E, Mourmouras V, Monciatti I, Mannucci S, Biagioli M, Toscano M, Moretti D, Lio R, Massi D. Beclin 1 and LC3 autophagic gene expression in cutaneous melanocytic lesions. Human pathology. 2010; 41:503-512.

10. Zhou S, Zhao L, Kuang M, Zhang B, Liang Z, Yi T, Wei Y, Zhao X. Autophagy in tumorigenesis and cancer therapy: Dr. Jekyll or Mr. Hyde? Cancer Lett. 2012; 323:115-127.

11. Zhu K, Dunner K, Jr. , McConkey DJ. Proteasome inhibitors activate autophagy as a cytoprotective response in human prostate cancer cells. Oncogene. 2010; 29:451-462.

12. Kim RH, Bold RJ , Kung HJ. ADI, autophagy and apoptosis: metabolic stress as a therapeutic option for prostate cancer. Autophagy. 2009; 5:567-568.

13. Saleem A, Dvorzhinski D, Santanam U, Mathew R, Bray K, Stein M, White E, Dipaola RS. Effect of dual inhibition of apoptosis and autophagy in prostate cancer. Prostate. 2012; 72:1374-1381.

14. Kung HJ. Targeting tyrosine kinases and autophagy in prostate cancer. Horm Cancer. 2011; 2:38-46.

15. Wu Z, Chang PC, Yang JC, Chu CY, Wang LY, Chen NT, Ma AH, Desai SJ, Lo SH, Evans CP, Lam KS , Kung HJ. Autophagy blockade sensitizes prostate cancer cells towards src family kinase inhibitors. Genes Cancer. 2010; 1:40-49 doi: $10.1177 / 1947601909358324$.
16. Li M, Jiang X, Liu D, Na Y, Gao GF, Xi Z. Autophagy protects $\mathrm{LNCaP}$ cells under androgen deprivation conditions. Autophagy. 2008; 4:54-60.

17. Bennett HL, Fleming JT, O'Prey J, Ryan KM , Leung HY. Androgens modulate autophagy and cell death via regulation of the endoplasmic reticulum chaperone glucoseregulated protein 78/BiP in prostate cancer cells. Cell Death Dis. 2010; 1:e72.

18. Levine B. Unraveling the role of autophagy in cancer. Autophagy. 2006; 2:65-66.

19. Baehrecke EH. Autophagy: dual roles in life and death? Nature reviews Molecular cell biology. 2005; 6:505-510.

20. Mathew R, Karp CM, Beaudoin B, Vuong N, Chen G, Chen HY, Bray K, Reddy A, Bhanot G, Gelinas C, Dipaola RS, Karantza-Wadsworth V, White E. Autophagy suppresses tumorigenesis through elimination of p62. Cell. 2009; 137:1062-1075.

21. Liu H, He Z, von Rutte T, Yousefi S, Hunger RE, Simon HU. Down-regulation of autophagy-related protein 5 (ATG5) contributes to the pathogenesis of early-stage cutaneous melanoma. Sci Transl Med. 2013; 5:202ra123.

22. Liang XH, Jackson S, Seaman M, Brown K, Kempkes B, Hibshoosh H , Levine B. Induction of autophagy and inhibition of tumorigenesis by beclin 1. Nature. 1999; 402:672-676.

23. Qu X, Yu J, Bhagat G, Furuya N, Hibshoosh H, Troxel A, Rosen J, Eskelinen EL, Mizushima N, Ohsumi Y, Cattoretti $\mathrm{G}$, Levine B. Promotion of tumorigenesis by heterozygous disruption of the beclin 1 autophagy gene. The Journal of clinical investigation. 2003; 112:1809-1820.

24. Degenhardt K, Mathew R, Beaudoin B, Bray K, Anderson D, Chen G, Mukherjee C, Shi Y, Gelinas C, Fan Y, Nelson DA, Jin S, White E. Autophagy promotes tumor cell survival and restricts necrosis, inflammation, and tumorigenesis. Cancer Cell. 2006; 10:51-64.

25. Giatromanolaki A, Sivridis E, Mendrinos S, Koutsopoulos AV, Koukourakis MI. Autophagy proteins in prostate cancer: relation with anaerobic metabolism and Gleason score. Urologic oncology. 2014; 32:39 e11-38.

26. Liu B, Miyake H, Nishikawa M, Tei H, Fujisawa M. Expression profile of autophagy-related markers in localized prostate cancer: correlation with biochemical recurrence after radical prostatectomy. Urology. 2015; 85:1424-1430.

27. Farrow JM, Yang JC, Evans CP. Autophagy as a modulator and target in prostate cancer. Nature reviews Urology. 2014; 11:508-516.

28. Koukourakis MI, Kalamida D, Mitrakas A, Pouliliou S, Kalamida S, Sivridis E , Giatromanolaki A. Intensified autophagy compromises the efficacy of radiotherapy against prostate cancer. Biochemical and biophysical research communications. 2015; 461:268-274.

29. Kononen J, Bubendorf L, Kallioniemi A, Barlund M, Schraml P, Leighton S, Torhorst J, Mihatsch MJ, Sauter G, 
Kallioniemi OP. Tissue microarrays for high-throughput molecular profiling of tumor specimens. Nature medicine. 1998; 4:844-847.

30. Epstein JI, Allsbrook WC, Jr., Amin MB, Egevad LL, Committee IG. The 2005 International Society of Urological Pathology (ISUP) Consensus Conference on Gleason Grading of Prostatic Carcinoma. The American journal of surgical pathology. 2005; 29:1228-1242.

31. Chittaranjan S, Bortnik S, Dragowska WH, Xu J, Abeysundara N, Leung A, Go NE, DeVorkin L, Weppler SA, Gelmon K, Yapp DT, Bally MB, Gorski SM. Autophagy inhibition augments the anticancer effects of epirubicin treatment in anthracycline-sensitive and -resistant triple-negative breast cancer. Clin Cancer Res. 2014; 20:3159-3173.

32. Qin L, Wang Z, Tao L and Wang Y. ER stress negatively regulates $\mathrm{AKT} / \mathrm{TSC} / \mathrm{mTOR}$ pathway to enhance autophagy. Autophagy. 2010; 6:239-247.
33. Huang Y, Hou JK, Chen TT, Zhao XY, Yan ZW, Zhang J, Yang J, Kogan SC, Chen GQ. PML-RARalpha enhances constitutive autophagic activity through inhibiting the Akt/ mTOR pathway. Autophagy. 2011; 7:1132-1144.

34. Ladoire S, Chaba K, Martins I, Sukkurwala AQ, Adjemian S, Michaud M, Poirier-Colame V, Andreiuolo F, Galluzzi L, White E, Rosenfeldt M, Ryan KM, Zitvogel L, et al. Immunohistochemical detection of cytoplasmic LC3 puncta in human cancer specimens. Autophagy. 2012; 8:1175-1184.

35. Petiot A, Ogier-Denis E, Blommaart EF, Meijer AJ, Codogno P. Distinct classes of phosphatidylinositol 3'-kinases are involved in signaling pathways that control macroautophagy in HT-29 cells. J Biol Chem. 2000; 275:992-998. 